\title{
Scalar Domain Wall as the Universe
}

\author{
Yuta Toyozato ${ }^{1, *}$, Kazuharu Bamba ${ }^{2, \dagger}$, Shin’ichi Nojiri ${ }^{1,2, \ddagger}$ \\ 1 Department of Physics, Nagoya University, Nagoya 464-8602, Japan \\ ${ }^{2}$ Kobayashi-Maskawa Institute for the Origin of Particles and the Universe, Nagoya University, Nagoya 464-8602, Japan
}

\begin{abstract}
We develop a formulation where for an arbitrarily given warp factor, we construct a scalar field action which reproduces the warp factor as a solution of the Einstein equation and field equation corresponding to the action. This formulation could be called as the reconstruction. By using the formulation of the reconstruction, we construct models which have an exact solution describing the domain wall. The shape of the domain wall can be flat, de Sitter space-time, or anti-de Sitter spacetime. In the constructed domain wall solutions, there often appears ghost with negative kinetic energy. We give, however, an example of the de Sitter domain wall solution without ghost, which could be a toy model of inflation. We also investigate the localization of gravity as in the RandallSundrum model. It is demonstrated that the four dimensional Newton law could be reproduced even in the de Sitter space-time domain wall solution. We show that we can construct a space-time, where the domain wall is the general Friedmann-Robertson-Walker (FRW) universe and the warp factor can be arbitrary. For such a construction, we use two scalar fields. It is also illustrated that the scalar field equations are equivalent to the Bianchi identities: $\nabla^{\mu}\left(R_{\mu \nu}-\frac{1}{2} R g_{\mu \nu}\right)=0$.
\end{abstract}

PACS numbers: 95.36.+x, 98.80.Cq

\section{INTRODUCTION}

There are many scenarios that our universe could be a brane in the higher dimensional space-time [1 5]. The inflationary brane world models were also considered by using the trace anomaly in [6 6 ] . Before the brane world scenario, there was a scenario that we live on the domain wall [9], and bent domain wall [10] as well as dynamical domain wall [11] were also investigated. After that there were many activities in the domain wall or thick brane universe scenario 12 17]. The domain wall has a thickness and the brane could be regarded as a limit that the thickness of the domain wall vanishes.

In this paper, we consider the domain wall by using the scalar field. We construct models where the shape of the domain wall is flat, de Sitter space-time, or anti-de Sitter space-time. In case that the shape of the domain wall is de Sitter space-time, if we regard the domain wall as our universe, the de Sitter space-time may express inflation and/or the accelerating expansion of the current universe. It is developed a formulation to construct an action which reproduces an arbitrarily given warp factor as a solution of the Einstein equation and field equation corresponding to the action. This formulation could be called as the reconstruction. By using this formulation ${ }^{1}$ we give models which have an exact solution describing the domain wall. In the seminal paper [12], a formulation of the reconstruction has been proposed. In case of the brane with vanishing thickness, the potential which realizes the arbitrary warp factor has been analytically found. In case of the domain wall or thick brane, we need to solve first order differential equations to find the potential. In the formulation which we propose, even for the domain wall, we only need algebraic calculations and the action of the model can be obtained straightforwardly. We also show that there appears massless graviton propagating on the four dimensional domain wall. The four dimensional massless graviton is the zero mode of the five dimensional graviton and the existence of the four dimensional massless graviton may tell that the four dimensional gravity, where the Newton potential behaves as $1 / r$ for the distance $r$, could be reproduced on the domain wall. Furthermore, we explore the models including two scalar fields and show that we can construct a space-time, where the domain wall is the general FRW universe and the warp factor can be arbitrary. The scalar field equations can be identified with the Bianchi identities: $\nabla^{\mu}\left(R_{\mu \nu}-\frac{1}{2} R g_{\mu \nu}\right)=0$ and therefore the equations can be satisfied automatically.

In the next section, we explain the formulation to obtain models which admit exact solutions describing the domain wall and we also give some examples. In the domain wall solutions, there appears ghost in general. The ghost has negative kinetic energy. We give, however, an example of de Sitter domain wall without ghost, which can be a toy model of inflation. In Section III we investigate the localization of gravity and show the four dimensional Newton

\footnotetext{
* E-mail address: toyozato@th.phys.nagoya-u.ac.jp

$\dagger$ E-mail address: bamba@kmi.nagoya-u.ac.jp

¥ E-mail address: nojiri@phys.nagoya-u.ac.jp

1 About the reconstruction for cosmology, see [18].
} 
law could be reproduced. In Section IV] we propose a two scalar model. By using the model, we show that we can construct a space-time, where the domain wall is the general FRW universe and the warp factor can be arbitrary. In Section V, we present examples of reconstructed model. The last section is devoted to the summary and discussion.

We use units of $k_{\mathrm{B}}=c=\hbar=1$ and denote the gravitational constant $8 \pi G$ by $\kappa^{2} \equiv 8 \pi / M_{\mathrm{Pl}}^{2}$ with the Planck mass of $M_{\mathrm{Pl}}=G^{-1 / 2}=1.2 \times 10^{19} \mathrm{GeV}$.

\section{CONSTRUCTING THE ACTION GENERATING AN EXACT DOMAIN WALL SOLUTION}

In this section, based on [19], we show how we can construct models which admit the exact solutions describing the domain wall. We use a procedure proposed in Ref. 20]. This formulation is a kind of reconstruction, that is, for an arbitrary warp factor, we specify the action of the Einstein gravity coupled with a scalar field which has the solution corresponding to the scale factor.

Our model action is as follows:

$$
S=\int d^{D} x \sqrt{-g}\left(\frac{R}{2 \kappa^{2}}-\frac{\omega(\varphi)}{2} \partial_{\mu} \varphi \partial^{\mu} \varphi-\mathcal{V}(\phi)\right)
$$

We assume the $D=d+1$ dimensional warped metric

$$
d s^{2}=d y^{2}+L^{2} \mathrm{e}^{u(y)} \sum_{\mu, \nu=0}^{d-1} \hat{g}_{\mu \nu} d x^{\mu} d x^{\nu}
$$

with $L$ being a dimensionless constant. We suppose that the scalar field only depends on $y$. In the metric (2.2), $\hat{g}_{\mu \nu}$ is the metric of the $d$-dimensional Einstein manifold defined by $\hat{R}_{\mu \nu}=\left[(d-1) / l^{2}\right] \hat{g}_{\mu \nu}$. The de Sitter space (the anti-de Sitter space) corresponds to $1 / l^{2}>0\left(1 / l^{2}<0\right)$, whereas the flat space to $1 / l^{2}=0$. The $(y, y)$ and $(\mu, \nu)$ components of the Einstein equation are given by

$$
\begin{aligned}
& -\frac{d(d-1)}{2 l^{2}} \mathrm{e}^{-u}+\frac{d(d-1)}{8}\left(u^{\prime}\right)^{2}=\frac{1}{2} \omega(\varphi)\left(\varphi^{\prime}\right)^{2}-\mathcal{V}(\varphi) \\
& -\frac{(d-1)(d-2)}{2 l^{2}} \mathrm{e}^{-u}+\frac{d-1}{2} u^{\prime \prime}+\frac{d(d-1)}{8}\left(u^{\prime}\right)^{2}=-\frac{1}{2} \omega(\varphi)\left(\varphi^{\prime}\right)^{2}-\mathcal{V}(\varphi)
\end{aligned}
$$

Here the prime denotes the derivative with respect to $y$. Now $\varphi$ may be chosen as $\varphi=y$. Moreover, we take $\kappa^{2}=1$. Then Eqs. (2.3) and (2.4) lead to

$$
\begin{aligned}
& \omega(\varphi)=-\frac{d-1}{2} u^{\prime \prime}-\frac{d-1}{l^{2}} \mathrm{e}^{-u} \\
& \mathcal{V}(\varphi)=-\frac{d-1}{4} u^{\prime \prime}-\frac{d(d-1)}{8}\left(u^{\prime}\right)^{2}+\frac{(d-1)^{2}}{2 l^{2}} \mathrm{e}^{-u}
\end{aligned}
$$

Hence, the energy density $\rho$ is now described as [19]

$$
\rho=\frac{\omega(\varphi)}{2}\left(\varphi^{\prime}\right)^{2}+\mathcal{V}(\varphi)=-\frac{d-1}{2} u^{\prime \prime}-\frac{d(d-1)}{8}\left(u^{\prime}\right)^{2}+\frac{(d-1)(d-2)}{2 l^{2}} \mathrm{e}^{-u} .
$$

The pressure $p_{y}$ for the $y$ direction and the pressures $p$ for other spatial directions are different with each other and given by

$$
\begin{aligned}
& p_{y}=\frac{\omega(\varphi)}{2}\left(\varphi^{\prime}\right)^{2}-\mathcal{V}(\varphi)=\frac{d(d-1)}{8}\left(u^{\prime}\right)^{2}-\frac{(d-1)(d-2)}{2 l^{2}} \mathrm{e}^{-u} \\
& p=\rho=-\frac{d-1}{2} u^{\prime \prime}-\frac{d(d-1)}{8}\left(u^{\prime}\right)^{2}+\frac{(d-1)(d-2)}{2 l^{2}} \mathrm{e}^{-u}
\end{aligned}
$$

Provided that the $D$ dimensional space is asymptotically flat, we see $u \rightarrow 0$ in the limit of $|y| \rightarrow \infty$, the second term becomes dominant in (2.5), $\omega(\varphi) \sim-(d-1) / l^{2}$ if $1 / l^{2} \neq 0$. If $\omega(\varphi)$ is negative, which corresponds to the de Sitter space with $1 / l^{2}>0$, the scalar field $\varphi$ is ghost. For $1 / l^{2}=0$, we obtain $\omega(\varphi)=-(d-1) u^{\prime \prime} / 2$. Thus we impose the $Z_{2}$ symmetry of the metric, which is the invariance under the transformation $y \rightarrow-y$. In addition, we suppose the $D$ dimensional space is asymptotically flat. In such a case, there must exist a region in which $\omega(\varphi)$ is negative and 
hence $\varphi$ is ghost. We should also remark that the energy density often becomes negative. In any case, if we allow the ghost and negative energy density, for an arbitrary $u$, we find a model which permits $u$ to be a solution of the Einstein equation. Furthermore, the problem of ghost can be avoided in case that the extra dimensions are compact.

As an example, we may examine

$$
u=u_{0} \mathrm{e}^{-y^{2} / y_{0}^{2}}
$$

where $u_{0}$ and $y_{0}$ are constants. We explore the model

$$
\begin{aligned}
& \omega(\varphi)=-(d-1)\left(\frac{2 \varphi^{2}}{y_{0}^{4}}-\frac{1}{y_{0}^{2}}\right) \mathrm{e}^{-\varphi^{2} / y_{0}^{2}}-\frac{(d-1)}{l^{2}} \mathrm{e}^{-u_{0} \mathrm{e}^{-\varphi^{2} / y_{0}^{2}}} \\
& \mathcal{V}(\varphi)=-\frac{d-1}{2}\left(\frac{2 \varphi^{2}}{y_{0}^{4}}-\frac{1}{y_{0}^{2}}\right) \mathrm{e}^{-\varphi^{2} / y_{0}^{2}}+\frac{(d-1)^{2}}{l^{2}} \mathrm{e}^{-u_{0} \mathrm{e}^{-\varphi^{2} / y_{0}^{2}}}
\end{aligned}
$$

In this model, as a solution of the Einstein equation we acquire $u$ in (2.9). Furthermore, we find the following distribution of the energy density [19]

$$
\rho(y)=-\frac{d-1}{2}\left(\frac{2 y^{2}}{y_{0}^{4}}-\frac{1}{y_{0}^{2}}\right) \mathrm{e}^{-y^{2} / y_{0}^{2}}+\frac{(d-1)^{2}}{l^{2}} \mathrm{e}^{-u_{0} \mathrm{e}^{-y^{2} / y_{0}^{2}}} .
$$

As a result, the energy density $\rho(y)$ is localized at $y \sim 0$ and therefore a domain wall is made. We also mention that for $1 / l^{2}>0$, the shape of the domain wall is a de Sitter space, and hence it could represent the accelerating universe.

\section{A. (Anti-)de Sitter space-time}

As a preparation to deal with the domain wall, here we consider the (anti-)de Sitter space-time and the flat spacetime. These space-times do not correspond to brane or domain wall but we give explicit formula for later use.

We now study the de Sitter space-time solution. When $L^{2}=l^{2}>0(L$ appears in $(2.2))$, if $u(y)$ is given by

$$
u=2 \ln \cosh \frac{y}{l}
$$

we find

$$
\omega(\varphi)=0, \quad \mathcal{V}(\varphi)=\frac{d(d-1)}{2 l^{2}}
$$

We may also investigate the anti-de Sitter solution. When $L^{2}=l^{2}>0$, if $u(y)$ is given by

$$
u=2 \ln \sinh \frac{y}{l},
$$

we acquire

$$
\omega(\varphi)=0, \quad \mathcal{V}(\varphi)=-\frac{d(d-1)}{2 l^{2}}
$$

Here $y$ is restricted to be $y \geq 0$. On the other hand, when $l^{2}=-\tilde{l}^{2}=-L^{2}<0$, if $u(y)$ is given by

$$
u=2 \ln \cosh \frac{y}{\tilde{l}},
$$

we have

$$
\omega(\varphi)=0, \quad \mathcal{V}(\varphi)=-\frac{d(d-1)}{2 \tilde{l}^{2}}
$$

When $1 / l^{2}=0$, if $u(y)$ is given by

$$
u=\frac{2 y}{L},
$$


where $L$ is a constant, we obtain

$$
\omega(\varphi)=0, \quad \mathcal{V}(\varphi)=-\frac{d(d-1)}{2 L^{2}}
$$

We now examine the flat space-time. When $l^{2}>0$, we find

$$
u=2 \ln \frac{y}{l}, \quad \omega(\varphi)=\mathcal{V}(\varphi)=0 .
$$

Here $y \geq 0$. When $1 / l^{2}=0$, we, of course, acquire

$$
u=\omega(\varphi)=\mathcal{V}(\varphi)=0 .
$$

\section{B. Randall-Sundrum like model}

In case of the second Randall-Sundrum model [2], we have

$$
1 / l^{2}=0, \quad u(y)=-\frac{2|y|}{L} .
$$

This model shows the localization of the gravity on the four dimensional brane. Motivated by the model (2.22), we analyze the following model,

$$
1 / l^{2}=0, \quad u(y)=-\frac{2 \sqrt{y^{2}+y_{0}^{2}}}{L}
$$

Here $y_{0}$ is a constant and the Randall-Sundrum model (2.22) corresponds to this model in the limit of $y_{0} \rightarrow 0$. Then we obtain

$$
\omega(\varphi)=\frac{(d-1) y_{0}^{2}}{L\left(\varphi^{2}+y_{0}^{2}\right)^{\frac{3}{2}}}, \quad \mathcal{V}(\varphi)=\frac{(d-1) y_{0}^{2}}{2 L\left(\varphi^{2}+y_{0}^{2}\right)^{\frac{3}{2}}}-\frac{d(d-1) \varphi^{2}}{2 L^{2}\left(\varphi^{2}+y_{0}^{2}\right)}
$$

Then the energy density is given by

$$
\rho(y)=\frac{(d-1) y_{0}^{2}}{L\left(y^{2}+y_{0}^{2}\right)^{\frac{3}{2}}}-\frac{d(d-1) y^{2}}{2 L^{2}\left(y^{2}+y_{0}^{2}\right)} .
$$

In the limit of $y_{0} \rightarrow 0$, the second term in (2.25) gives the negative cosmological constant corresponding to the anti-de Sitter space-time and the first term gives a $\delta$-function:

$$
\frac{(d-1) y_{0}^{2}}{L\left(y^{2}+y_{0}^{2}\right)^{\frac{3}{2}}} \rightarrow \frac{2(d-1)}{L} \delta(y), \quad-\frac{d(d-1) y^{2}}{2 L^{2}\left(y^{2}+y_{0}^{2}\right)} \rightarrow-\Lambda \equiv-\frac{d(d-1)}{2 L^{2}} .
$$

On the other hand, $p_{y}$ and $p$ in (2.8) are given by

$$
p_{y}=\frac{d(d-1) y^{2}}{2 L^{2}\left(y^{2}+y_{0}^{2}\right)} \rightarrow \Lambda=\frac{d(d-1)}{2 L^{2}}, \quad p=\rho \rightarrow \frac{2(d-1)}{L} \delta(y)-\frac{d(d-1)}{2 L^{2}} .
$$

\section{C. de Sitter domain wall and brane}

We now explore the de Sitter domain wall and brane with $l^{2}>0$, which may correspond to the expanding universe. The four dimensional de Sitter brane solution in five dimensional anti-de Sitter space-time using the trace anomaly is given by [6 8].

Motivated by (2.14), we may study

$$
u=2 \sinh \left(\frac{\sqrt{y_{0}^{2}+z_{0}^{2}}-\sqrt{y_{0}^{2}+z^{2}}}{l^{\prime}}\right), \quad l^{\prime} \equiv \frac{l z_{0}}{\sqrt{y_{0}^{2}+z_{0}^{2}}} .
$$


Here $y_{0}$ and $z_{0}$ are positive constants and we assume $-z_{0} \leq z \leq z_{0}$. In the limit of $y_{0} \rightarrow 0$, we find

$$
\sqrt{y_{0}^{2}+z_{0}^{2}}-\sqrt{y_{0}^{2}+z^{2}} \rightarrow z_{0}-|z|
$$

which implies that the space-time given by (2.28) in this limit can be obtained as follows: First cut two anti-de Sitter spaces given by (2.14) at $y=z_{0}$. Second glue two space-times with the region $0 \leq y \leq z_{0}$ at $y=z_{0}$. Then in the limit (2.29), $z=0$ corresponds to $y=z_{0}$ and $z= \pm z_{0}$ to $y=0$. Then we find

$$
\begin{aligned}
\omega(\varphi)= & (d-1)\left(\frac{\frac{\varphi^{2}}{l^{2}\left(\varphi^{2}+y_{0}^{2}\right)}}{\sinh ^{2}\left(\frac{\sqrt{y_{0}^{2}+z_{0}^{2}}-\sqrt{y_{0}^{2}+\varphi^{2}}}{l^{\prime}}\right)}-\frac{y_{0}^{2}}{l^{\prime}\left(\varphi^{2}+y_{0}^{2}\right)^{\frac{3}{2}}} \frac{\cosh \left(\frac{\sqrt{y_{0}^{2}+z_{0}^{2}}-\sqrt{y_{0}^{2}+\varphi^{2}}}{l^{\prime}}\right)}{\sinh \left(\frac{\sqrt{y_{0}^{2}+z_{0}^{2}}-\sqrt{y_{0}^{2}+\varphi^{2}}}{l^{\prime}}\right)}\right) \\
& -\frac{d-1}{l^{2} \sinh ^{2}\left(\frac{\sqrt{y_{0}^{2}+z_{0}^{2}}-\sqrt{y_{0}^{2}+\varphi^{2}}}{l^{\prime}}\right)}, \\
\mathcal{V}(\varphi)= & \frac{(d-1)}{2}\left(\frac{\frac{\varphi^{2}}{l^{\prime 2}\left(\varphi^{2}+y_{0}^{2}\right)}}{\sinh ^{2}\left(\frac{\sqrt{y_{0}^{2}+z_{0}^{2}}-\sqrt{y_{0}^{2}+\varphi^{2}}}{l^{\prime}}\right)}-\frac{y_{0}^{2}}{l^{\prime}\left(\varphi^{2}+y_{0}^{2}\right)^{\frac{3}{2}}} \frac{\cosh \left(\frac{\sqrt{y_{0}^{2}+z_{0}^{2}}-\sqrt{y_{0}^{2}+\varphi^{2}}}{l^{\prime}}\right)}{\sinh \left(\frac{\sqrt{y_{0}^{2}+z_{0}^{2}}-\sqrt{y_{0}^{2}+\varphi^{2}}}{l^{\prime}}\right)}\right) \\
& -\frac{d(d-1)}{2} \frac{\varphi^{2}}{l^{\prime 2}\left(\varphi^{2}+y_{0}^{2}\right)} \frac{\cosh ^{2}\left(\frac{\sqrt{y_{0}^{2}+z_{0}^{2}}-\sqrt{y_{0}^{2}+\varphi^{2}}}{l^{\prime}}\right)}{\sinh ^{2}\left(\frac{\sqrt{y_{0}^{2}+z_{0}^{2}}-\sqrt{y_{0}^{2}+\varphi^{2}}}{l^{\prime}}\right)} \\
& +\frac{d-1}{2 l^{2} \sinh ^{2}\left(\frac{\sqrt{y_{0}^{2}+z_{0}^{2}}-\sqrt{y_{0}^{2}+\varphi^{2}}}{l^{\prime}}\right)} .
\end{aligned}
$$

Here we have identified $z$ as $\varphi$. We should note that $\omega(\varphi)$ is not always positive and hence the scalar field becomes ghost.

We now show that we can construct a model without ghost. For simplicity, we only examine the case $D=d+1=5$. Let us explore the following model:

$$
\mathrm{e}^{u(y)}=\frac{y^{2}}{l^{2}}\left(1+\frac{y^{4}}{y_{0}^{4}}\right)^{-1}
$$

When $y \rightarrow 0, \mathrm{e}^{u(y)}$ behaves as $\mathrm{e}^{u(y)} \sim y^{2} / l^{2}$. Therefore we can regard $y$ as a radial coordinate and $y=0$ corresponds to the center of the manifold whose topology of the spatial part is a four dimensional sphere. Since $\left.d\left(\mathrm{e}^{u(y)}\right)\right|_{y=y_{0}}=0$, we can cut the manifold at $y=y_{0}$ and glue two copies of the manifold cut at $y=y_{0}$. For the model (2.31), we find

$$
\begin{aligned}
& \omega(\varphi)=3\left(1+\frac{\varphi^{4}}{y_{0}^{4}}\right)^{-2} \frac{\varphi^{2}}{y_{0}^{4}}\left(5+\frac{\varphi^{4}}{y_{0}^{4}}\right)\left(1-\frac{\varphi^{4}}{y_{0}^{4}}\right), \\
& \mathcal{V}(\varphi)=\left(1+\frac{\varphi^{4}}{y_{0}^{4}}\right)^{-2}\left(\frac{75 \varphi^{2}}{2 y_{0}^{4}}+\frac{6 \varphi^{6}}{y_{0}^{8}}+\frac{9 \varphi^{10}}{2 y_{0}^{12}}\right) .
\end{aligned}
$$

When $y^{2}=\varphi^{2} \leq y_{0}^{2}$, both $\omega(\varphi)$ and $\mathcal{V}(\varphi)$ are positive and therefore there does not appear ghost. In order to avoid the ghost, we may restrict the value of $\varphi$ to be $\varphi^{2} \leq y_{0}^{2}$ The energy density is also given by

$$
\rho(y)=\left(1+\frac{y^{4}}{y_{0}^{4}}\right)^{-2}\left(\frac{45 y^{2}}{y_{0}^{4}}+\frac{3 y^{10}}{y_{0}^{12}}\right)
$$

which vanishes at $y=0$ and localizes at $y=y_{0}$ Then we have constructed a model of the de Sitter domain wall without ghost.

We may remark that, when we glue the two copies of the region $y^{2} \leq y_{0}^{2}$, the value of $d^{3} u / d y^{3}$ becomes discontinuous at $y=y_{0}$ although the values of $u, u^{\prime}$, and $u^{\prime \prime}$ are continuous. This means that the derivative of the curvatures with respect to $y$ and therefore the derivative of the energy density in (2.33) become discontinuous at $y=y_{0}$. We should note, however, that this discontinuities never conflicts with the Einstein equation and field equation since these equations do not contain the derivative higher than two. 


\section{LOCALIZATION OF GRAVITY}

As executed in [2], we now investigate the localization of the gravity. Here we restrict the consideration to the case of $d=4$ for simplicity. For this purpose, we examine the following perturbation

$$
g_{\mu \nu}=g_{\mu \nu}^{(0)}+h_{\mu \nu} .
$$

Here $g_{\mu \nu}^{(0)}$ is the metric given in the previous sections by solving the Einstein equations, etc. We express the quantities given by $g_{\mu \nu}^{(0)}$ by using the suffix (0) and we define the lowering and raising of the vector index by using $g_{\mu \nu}^{(0)}$ and $g^{(0) \mu \nu}$ like $h^{\mu}{ }_{\nu}=g^{(0) \mu \rho} h_{\rho \nu}$.

We have

$$
\begin{aligned}
\sqrt{-g}= & \sqrt{-g^{(0)}}\left(1+\frac{1}{2} h_{\mu}^{\mu}+\frac{1}{8}\left(h_{\mu}^{\mu}\right)^{2}-\frac{1}{4} h_{\mu \nu} h^{\mu \nu}+\mathcal{O}\left(h^{3}\right)\right) \\
R= & R^{(0)}-R^{(0) \mu \nu} h_{\mu \nu}+\nabla^{(0) \mu} \nabla^{(0) \nu} h_{\mu \nu}-\nabla^{(0)^{2}} h_{\mu}^{\mu} \\
& +\frac{3}{4}\left(\nabla^{(0) \sigma} h_{\rho}^{\rho}\right) \nabla^{(0) \nu} h_{\sigma \nu}+\frac{3}{4} h^{\mu \nu} \nabla_{\mu}^{(0)} \nabla_{\nu}^{(0)} h_{\rho}{ }^{\rho}-\frac{1}{2} \nabla^{(0) \mu} h_{\mu \lambda} \nabla_{\nu}^{(0)} h^{\nu \lambda} \\
& +\frac{1}{4} h^{\mu \nu} \nabla^{(0)^{2}} h_{\mu \nu}-\frac{1}{4}\left(\nabla^{(0) \sigma} h_{\rho}^{\rho}\right)\left(\nabla_{\sigma}^{(0)} h_{\mu}^{\mu}\right)+\frac{1}{2} R_{\mu \nu}^{(0)} h^{\mu \rho} h_{\rho}^{\nu}+\frac{1}{2} R^{(0) \rho \mu \sigma \nu} h_{\rho \sigma} h_{\mu \nu} \\
& +\nabla^{(0) \mu}\left(-\frac{1}{4} h_{\kappa \nu} \nabla_{\mu} h^{\nu \kappa}-\frac{1}{2} h_{\mu \kappa} \nabla_{\rho}^{(0)} h^{\rho \kappa}+\frac{1}{4} h_{\mu \kappa} \nabla^{(0) \kappa} h_{\rho}^{\rho}\right) \\
& +\frac{1}{2} \nabla^{(0)^{2}}\left(h_{\mu \nu} h^{\mu \nu}\right)+\mathcal{O}\left(h^{3}\right) .
\end{aligned}
$$

Since we are interested in the localization of the graviton, which is massless and a spin two particle, we now assume,

$$
h_{0 \mu}=h_{y \mu}=\nabla^{(0) i} h_{i j}=h_{i}^{j}=0 .
$$

In the following, we only study $D=d+1=5$ case for simplicity. Thus the action is reduced as follows:

$$
\begin{aligned}
& \int d^{5} x \sqrt{-g}\left[\frac{R}{2 \kappa^{2}}-\frac{1}{2} \omega(\varphi) \partial_{\mu} \varphi \partial^{\mu} \varphi-\mathcal{V}(\varphi)\right] \\
& \rightarrow \int d^{D} x \sqrt{-g^{(0)}}\left[\frac { 1 } { 2 \kappa ^ { 2 } } \left\{R^{(0)}-\frac{1}{4} \nabla^{(0) \rho} h^{i j} \nabla_{\rho}^{(0)} h_{i j}+\frac{1}{2} R^{(0) i j} h_{i k} h_{j}{ }^{k}+\frac{1}{2} R^{(0) i k j l} h_{i j} h_{k l}\right.\right. \\
& \left.\left.\quad-\frac{1}{4} R^{(0)} h_{i j} h^{i j}\right\}-\frac{1}{4} h_{i j} h^{i j}\left(-\frac{1}{2} \omega(\varphi) \partial_{\mu} \varphi \partial^{\mu} \varphi-\mathcal{V}(\varphi)\right)+\mathcal{O}\left(h^{3}\right)\right]
\end{aligned}
$$

Then by the variation of $h_{i j}$, we obtain

$$
\begin{aligned}
0= & \frac{1}{2 \kappa^{2}}\left\{\frac{1}{2} \nabla^{\rho} \nabla_{\rho} h_{i j}+\frac{1}{2}\left(R_{i k}^{(0)} h^{k}{ }_{j}+R_{j k}^{(0)} h^{k}{ }_{i}\right)\right. \\
& \left.+R_{i k j l}^{(0)} h^{k l}-\frac{1}{2} R^{(0)} h_{i j}\right\}-\frac{1}{2 \kappa^{2}} h_{i j}\left(-\frac{1}{2} \omega(\varphi) \partial_{\mu} \varphi \partial^{\mu} \varphi-\mathcal{V}(\varphi)\right) .
\end{aligned}
$$

First we explore the case that the domain wall is flat, i.e., $\hat{g}_{\mu \nu}=\eta_{\mu \nu}$ in (2.2). Since

$$
\nabla^{\rho} \nabla_{\rho} h_{i j}=\left(\partial_{y}^{2}+\mathrm{e}^{-u} \square\right) h_{i j}-\left(u^{\prime \prime}+\frac{3}{2}{u^{\prime}}^{2}\right) h_{i j},
$$

we find

$$
0=\left(\partial_{y}^{2}+\mathrm{e}^{-u} \square\right) h_{i j}+\left(-u^{\prime \prime}-u^{\prime 2}\right) h_{i j} .
$$

Here we have used (2.5) and (2.6) with $d=4$. We should note that there is always a zero-mode solution, where $\square h_{i j}=0$ in the flat (domain wall) space-time. The explicit form of the zero-mode is given by

$$
h_{i j} \propto \mathrm{e}^{u},
$$


which could surely be normalizable if $u$ sufficiently rapidly goes to minus infinity $u \rightarrow+\infty$ when $|y| \rightarrow \infty$.

Next we investigate the case that the domain wall is de Sitter or anti-de Sitter space-time, namely, $\hat{R}_{\mu \nu}=\frac{3}{l^{2}} \hat{g}_{\mu \nu}$. Then instead of (3.7), we obtain

$$
0=\left(\partial_{y}^{2}+\mathrm{e}^{-u} \square\right) h_{i j}+\left(-u^{\prime \prime}-u^{\prime 2}-\frac{2 \mathrm{e}^{-u}}{l^{2}}\right) h_{i j} .
$$

Here we have used (2.5) and (2.6) with $d=4$. In the (anti)-de Sitter space-time, the zero-mode solution, which corresponds to the massless graviton, is given by

$$
\square h_{i j}=\frac{2}{l^{2}} h_{i j} .
$$

Therefore the zero-mode is again given by (3.8). Accordingly the zero-mode is always proportional to the warp factor $\mathrm{e}^{u}$. As a result, even in the (anti-)de Sitter domain wall, there occurs the localization of graviton.

\section{RECONSTRUCTION OF GENERAL FRW DOMAIN WALL UNIVERSE}

In this section, as an extension of the previous sections, we examine more general domain wall, which can be regarded as a general FRW universe. The metric we are considering is given by

$$
\begin{aligned}
d s^{2} & =d w^{2}+f(w, t)\left\{\frac{d r^{2}}{1-k r^{2}}+r^{2} d \theta^{2}+r^{2} \sin ^{2} \theta d \phi^{2}\right\}-\frac{e(w, t)^{2}}{f(w, t)} d t^{2} \\
& \equiv \mathrm{e}^{\ln f(w, t)} \gamma_{m n}(x) d x^{m} d x^{n}+h_{\alpha \beta}(y) d y^{\alpha} d y^{\beta} .
\end{aligned}
$$

Here $m, n=1,2,3, \alpha, \beta=0,5$, and $y^{0}=t, y^{5}=w$. Especially if we choose

$$
f(w, t)=L^{2} \mathrm{e}^{u(w, t)} a(t)^{2}, \quad e(w, t)=L^{2} \mathrm{e}^{u(w, t)} a(t),
$$

the general FRW universe, whose metric is described by

$$
d s_{\mathrm{FRW}}^{2}=-d t^{2}+a(t)^{2}\left\{\frac{d r^{2}}{1-k r^{2}}+r^{2} d \theta^{2}+r^{2} \sin ^{2} \theta d \phi^{2}\right\}
$$

is embedded by the arbitrary warp factor $L^{2} \mathrm{e}^{u(w, t)}$.

For the metric (4.1), the connections can be given by

$$
\hat{\Gamma}_{a b}^{m}=\Gamma_{a b}^{m}(\gamma), \quad \hat{\Gamma}_{n \alpha}^{m}=\hat{\Gamma}_{\alpha n}^{m}=\frac{1}{2} \delta_{n}^{m} \frac{\partial_{\alpha} f}{f}, \quad \hat{\Gamma}_{\beta \gamma}^{\alpha}=\Gamma_{\beta \gamma}^{\alpha}(h), \quad \hat{\Gamma}_{m n}^{\alpha}=-\frac{1}{2} \gamma_{m n} h^{\alpha \beta} \partial_{\beta} f,
$$

that is,

$$
\begin{aligned}
& \Gamma_{11}^{1}=\frac{k r}{1-k r^{2}}, \quad \Gamma_{12}^{2}=\frac{1}{r}, \quad \Gamma^{3}{ }_{13}=\frac{1}{r}, \quad \Gamma^{1}{ }_{22}=-r\left(1-k r^{2}\right), \\
& \Gamma_{23}^{3}=\cot \theta, \quad \Gamma_{33}^{1}=-r\left(1-k r^{2}\right) \sin ^{2} \theta, \quad \Gamma^{2}{ }_{33}=-\cos \theta \sin \theta, \\
& \Gamma_{00}^{0}=-\frac{e \dot{f}-2 \dot{e} f}{2 e f}, \quad \Gamma_{00}^{5}=-\frac{e^{2} f^{\prime}-2 e e^{\prime} f}{2 f^{2}}, \quad \Gamma_{05}^{0}=-\frac{e f^{\prime}-2 e^{\prime} f}{2 e f} .
\end{aligned}
$$

Then the non-vanishing Riemann tensors are expressed by

$$
\begin{aligned}
\hat{R}_{b c d}^{a} & ={ }^{(3)} R_{b c d}^{a}(\gamma)-\frac{1}{4}\left(\delta_{c}^{a} \gamma_{d b}-\delta_{d}^{a} \gamma_{c b}\right)\left\{\left(f^{\prime}\right)^{2}-\left(\frac{\dot{f}}{e^{2}}\right)^{2}\right\}, \\
\hat{R}_{\gamma b \delta}^{a} & =-\frac{1}{2} \delta_{b}^{a} \nabla_{\delta}^{(h)} \nabla_{\gamma}^{(h)} \ln f-\frac{1}{4} \delta_{b}^{a} \nabla_{\gamma}^{(h)} \ln f \nabla_{\delta}^{(h)} \ln f \\
\hat{R}_{b c \delta}^{\alpha} & =\frac{1}{2} \gamma_{c b} \nabla_{\delta}^{(h)}\left(h^{\alpha \kappa} \nabla_{\kappa}^{(h)} f\right)-\frac{1}{4} g_{c b} f h^{\alpha \kappa} \frac{\partial_{\kappa} f \partial_{\delta} f}{f} \\
\hat{R}_{\beta \gamma \delta}^{\alpha} & ={ }^{(2)} R_{\beta \gamma \delta}^{\alpha}(h)
\end{aligned}
$$


where

$$
\begin{aligned}
& { }^{(3)} R_{121}^{2}={ }^{(3)} R^{3}{ }_{131}=\frac{k}{1-k r^{2}}, \quad{ }^{(3)} R_{221}^{1}=-{ }^{(3)} R_{232}^{3}=-k r^{2}, \quad{ }^{(3)} R_{331}^{1}={ }^{(3)} R_{332}^{2}=-k r^{2} \sin ^{2} \theta, \\
& { }^{(2)} R_{050}^{5}=\frac{e^{2}}{f}{ }^{(2)} R_{550}^{0}=-\frac{2 e^{2} f f^{\prime \prime}-3 e^{2}\left(f^{\prime}\right)^{2}+4 e e^{\prime} f f^{\prime}-4 e e^{\prime \prime} f^{2}}{4 f^{3}} .
\end{aligned}
$$

Then the Einstein tensors have the following forms:

$$
\begin{aligned}
& G_{0}^{0}=\frac{3 f^{\prime \prime}}{2 f}-\frac{3 k}{f}-\frac{3 \dot{f}^{2}}{4 e^{2} f}, \\
& G_{1}^{1}=G_{2}^{2}=G_{3}^{3}=\frac{f^{\prime \prime}}{2 f}+\frac{e^{\prime \prime}}{e}-\frac{k}{f}-\frac{\ddot{f}}{e^{2}}-\frac{\dot{f}^{2}}{4 e^{2} f}+\frac{\dot{e} \dot{f}}{e^{3}}, \\
& G_{5}^{5}=\frac{3 f^{\prime} e^{\prime}}{2 f e}-\frac{3 k}{f}-\frac{3 \ddot{f}}{2 e^{2}}-\frac{3 \dot{f}^{2}}{4 e^{2} f}+\frac{3 \dot{e} \dot{f}}{2 e^{3}}, \\
& G_{0}^{5}=-\frac{3 e^{\prime} \dot{f}}{2 e^{3}}+\frac{3 \dot{f}^{\prime}}{2 e^{2}} .
\end{aligned}
$$

We now investigate the action of the scalar fields $\phi$ and $\chi$ :

$$
S_{\phi \chi}=\int d^{4} x \sqrt{-g}\left\{-\frac{1}{2} A(\phi, \chi) \partial_{\mu} \phi \partial^{\mu} \phi-B(\phi, \chi) \partial_{\mu} \phi \partial^{\mu} \chi-\frac{1}{2} C(\phi, \chi) \partial_{\mu} \chi \partial^{\mu} \chi-V(\phi, \chi)\right\} .
$$

We here construct a model to realize the arbitrary metric which can be written in the form of (4.1).

For the model (4.9), the energy-momentum tensor could be given by

$$
\begin{aligned}
T_{\mu \nu}^{\phi \chi}= & g_{\mu \nu}\left\{-\frac{1}{2} A(\phi, \chi) \partial_{\rho} \phi \partial^{\rho} \phi-B(\phi, \chi) \partial_{\rho} \phi \partial^{\rho} \chi-\frac{1}{2} C(\phi, \chi) \partial_{\rho} \chi \partial^{\rho} \chi-V(\phi, \chi)\right\} \\
& +A(\phi, \chi) \partial_{\mu} \phi \partial_{\nu} \phi+B(\phi, \chi)\left(\partial_{\mu} \phi \partial_{\nu} \chi+\partial_{\nu} \phi \partial_{\mu} \chi\right)+C(\phi, \chi) \partial_{\mu} \chi \partial_{\nu} \chi
\end{aligned}
$$

On the other hand, the field equations read

$$
\begin{aligned}
& 0=\frac{1}{2} A_{\phi} \partial_{\mu} \phi \partial^{\mu} \phi+A \nabla^{\mu} \partial_{\mu} \phi+A_{\chi} \partial_{\mu} \phi \partial^{\mu} \chi+\left(B_{\chi}-\frac{1}{2} C_{\phi}\right) \partial_{\mu} \chi \partial^{\mu} \chi+B \nabla^{\mu} \partial_{\mu} \chi-V_{\phi}, \\
& 0=\left(-\frac{1}{2} A_{\chi}+B_{\phi}\right) \partial_{\mu} \phi \partial^{\mu} \phi+B \nabla^{\mu} \partial_{\mu} \phi+\frac{1}{2} C_{\chi} \partial_{\mu} \chi \partial^{\mu} \chi+C \nabla^{\mu} \partial_{\mu} \chi+C_{\phi} \partial_{\mu} \phi \partial^{\mu} \chi-V_{\chi} .
\end{aligned}
$$

Here $A_{\phi}=\partial A(\phi, \chi) / \partial \phi$, etc. We now choose $\phi=t$ and $\chi=w$. Then we find

$$
T_{0}{ }^{0}=-\frac{f}{2 e^{2}} A-\frac{1}{2} C-V, \quad T_{i}{ }^{j}=\delta_{i}{ }^{j}\left(\frac{f}{2 e^{2}} A-\frac{1}{2} C-V\right), \quad T_{5}{ }^{5}=\frac{f}{2 e^{2}} A+\frac{1}{2} C-V, \quad T_{0}{ }^{5}=B,
$$

and

$$
\begin{aligned}
& 0=-\frac{f}{2 e^{2}} A_{\phi}+\frac{f}{e^{2}}\left(\frac{\dot{e}}{e}-\frac{2 \dot{f}}{f}\right) A+B_{\chi}+B\left(\frac{e^{\prime}}{e}+\frac{f^{\prime}}{f}\right)-\frac{1}{2} C_{\phi}-V_{\phi}, \\
& 0=\frac{f}{2 e^{2}} A_{\chi}-\frac{f}{e^{2}} B_{\phi}+\frac{f}{e^{2}}\left(\frac{\dot{e}}{e}-\frac{2 \dot{f}}{f}\right) B+\frac{1}{2} C_{\chi}+C\left(\frac{e^{\prime}}{e}+\frac{f^{\prime}}{f}\right)-V_{\chi} .
\end{aligned}
$$


Eqs. (4.13) can be solved with respect to $A, B, C$, and $V$ as follows,

$$
\begin{aligned}
A= & \frac{e^{2}}{\kappa^{2} f}\left(G_{1}{ }^{1}-G_{0}{ }^{0}\right)=\frac{e^{2}}{\kappa^{2} f}\left(G_{2}{ }^{2}-G_{0}{ }^{0}\right)=\frac{e^{2}}{\kappa^{2} f}\left(G_{3}{ }^{3}-G_{0}{ }^{0}\right) \\
= & \frac{1}{\kappa^{2}}\left(-\frac{e^{2} f^{\prime \prime}}{f^{2}}+\frac{e e^{\prime \prime}}{f}+\frac{2 k e^{2}}{f^{2}}-\frac{\ddot{f}}{f}+\frac{\dot{f}^{2}}{2 f^{2}}+\frac{\dot{e} \dot{f}}{e f}\right) \\
B= & \frac{1}{\kappa^{2}} G_{0}{ }^{5}=\frac{1}{\kappa^{2}}\left(-\frac{3 e^{\prime} \dot{f}}{2 e^{3}}+\frac{3 \dot{f}^{\prime}}{2 e^{2}}\right) \\
C= & \frac{1}{\kappa^{2}}\left(G_{5}{ }^{5}-G_{1}{ }^{1}\right)=\frac{1}{\kappa^{2}}\left(G_{5}{ }^{5}-G_{2}{ }^{1}\right)=\frac{1}{\kappa^{2}}\left(G_{5}{ }^{5}-G_{3}{ }^{1}\right) \\
= & \frac{1}{\kappa^{2}}\left(-\frac{f^{\prime \prime}}{2 f}-\frac{e^{\prime \prime}}{e}-\frac{2 k}{f}-\frac{\ddot{f}}{2 e^{2}}-\frac{\dot{f}^{2}}{2 e^{2} f}+\frac{\dot{e} \dot{f}}{2 e^{3}}+\frac{3 f^{\prime} e^{\prime}}{2 f e}\right) \\
V= & \frac{1}{\kappa^{2}}\left(G_{0}{ }^{0}+G_{5}{ }^{5}\right) \\
& \frac{1}{\kappa^{2}}\left(-\frac{3 f^{\prime \prime}}{4 f}+\frac{3 k}{f}+\frac{3 \dot{f}^{2}}{4 e^{2} f}-\frac{3 f^{\prime} e^{\prime}}{4 f e}+\frac{3 \ddot{f}}{4 e^{2}}-\frac{3 \dot{e} \dot{f}}{4 e^{3}}\right) .
\end{aligned}
$$

Then we find that the field equations (4.14) and (4.15) are nothing but the Bianchi identities:

$$
\begin{aligned}
& -\frac{f}{2 e^{2}} A_{\phi}+\frac{f}{e^{2}}\left(\frac{\dot{e}}{e}-\frac{2 \dot{f}}{f}\right) A+B_{\chi}+B\left(\frac{e^{\prime}}{e}+\frac{f^{\prime}}{f}\right)-\frac{1}{2} C_{\phi}-V_{\phi}=-\frac{e^{2}}{2 f} \nabla^{\mu} G_{\mu}{ }^{0}, \\
& \frac{f}{2 e^{2}} A_{\chi}-\frac{f}{e^{2}} B_{\phi}+\frac{f}{e^{2}}\left(\frac{\dot{e}}{e}-\frac{2 \dot{f}}{f}\right) B+\frac{1}{2} C_{\chi}+C\left(\frac{e^{\prime}}{e}+\frac{f^{\prime}}{f}\right)-V_{\chi}=\nabla^{\mu} G_{\mu}^{5} .
\end{aligned}
$$

Therefore the field equations (4.14) and (4.15) are surely satisfied by choosing $A, B, C$, and $V$ by (4.16).

Thus we can construct a model, where the general FRW universe can be embedded in an arbitrary warp factor, by the choice of $A, B, C$, and $V$ in (4.16).

\section{EXAMPLES OF RECONSTRUCTED MODEL}

In this section, we show some examples of reconstruction by using two scalar fields, where no ghost field appears. For simplicity, we consider examples in case $k=0$.

As a first example, we assume $a(t) \propto t^{h_{0}}$ with a constant $h_{0}$. Then, the equations in (4.16) give,

$$
\begin{aligned}
\kappa^{2} A & =-\frac{\ddot{U}}{U}+\frac{3 \dot{U}^{2}}{2 U^{2}}+\frac{h_{0} \dot{U}}{t U}+\frac{2 h_{0}}{t^{2}} \\
L^{2} \kappa^{2} B & =-\frac{3 U^{\prime} \dot{U}}{2 U^{3}}+\frac{3 \dot{U}^{\prime}}{2 U^{2}} \\
\kappa^{2} C & =-\frac{3 U^{\prime \prime}}{2 U}+\frac{3 U^{\prime 2}}{2 U^{2}}+\frac{1}{L^{2}}\left(-\frac{\ddot{U}}{2 U^{2}}-\frac{5 h_{0} \dot{U}}{2 t U^{2}}-\frac{3 h_{0}^{2}-h_{0}}{t^{2} U}\right) .
\end{aligned}
$$

Here $U(w, t) \equiv \mathrm{e}^{u(w, t)}$. Furthermore by assuming $U(w, t)=W(w) T(t)$, we rewrite (5.1) as follows,

$$
\begin{aligned}
\kappa^{2} A & =-\frac{\ddot{T}}{T}+\frac{3 \dot{T}^{2}}{2 T^{2}}+\frac{h_{0} \dot{T}}{t T}+\frac{2 h_{0}}{t^{2}} \\
L^{2} \kappa^{2} B & =0 \\
\kappa^{2} C & =-\frac{3 W^{\prime \prime}}{2 W}+\frac{3 W^{\prime 2}}{2 W^{2}}+\frac{1}{L^{2} W T^{2}}\left(-\frac{\ddot{T}}{2}-\frac{5 h_{0} \dot{T}}{2 t}-\frac{\left(3 h_{0}^{2}-h_{0}\right) T}{t^{2}}\right) .
\end{aligned}
$$


If we assume $T \propto t^{\beta}$, we find

$$
\begin{aligned}
-\frac{\ddot{T}}{2}-\frac{5 h_{0} \dot{T}}{2 t}-\frac{\left(3 h_{0}^{2}-h_{0}\right) T}{t^{2}} & \propto-\frac{1}{2}\left(\beta^{2}-\left(1-5 h_{0}\right) \beta+6 h_{0}^{2}-2 h_{0}\right) \\
& =-\frac{1}{2}\left\{\beta+\left(3 h_{0}-1\right)\right\}\left\{\beta+2 h_{0}\right\} .
\end{aligned}
$$

Then $T$ is given by

$$
T(t)=T_{1} t^{1-3 h_{0}}+T_{2} t^{-2 h_{0}}
$$

Therefore we obtain

$$
A=\frac{1}{\kappa^{2}}\left\{\frac{3}{2}\left(\frac{\dot{T}(t)}{T(t)}+\frac{2 h_{0}}{t}\right)^{2}\right\}>0, \quad \kappa^{2} C=-\frac{3 W^{\prime \prime}}{2 W}+\frac{3 W^{\prime 2}}{2 W^{2}}
$$

We may choose

$$
W(w)=\mathrm{e}^{-\frac{w^{2}}{w_{0}^{2}}}
$$

with a constant $w_{0}$, then we find

$$
C=\frac{3}{\kappa^{2} w_{0}^{2}}>0
$$

Because both of $A$ and $C$ are positive and $B$ vanishes, any ghost does not appear in this model. Since $a(t) \propto t^{h_{0}}$, the domain universe corresponds to the universe filled with the perfect fluid whose equation of state parameter $w$ is given by

$$
w=-1+\frac{2}{3 h_{0}} .
$$

Since we now have $f(w, t)=L^{2} T(t) \mathrm{e}^{-\omega^{2} / \omega_{0}^{2}} a_{0}^{2} t^{2 h_{0}}, e(w, t)=L^{2} T(t) \mathrm{e}^{-\omega^{2} / \omega_{0}^{2}} a_{0} t^{h_{0}}$ in (4.2). Then by using the last equation in (4.16), we find the explicit form of the potential $V$ :

$$
\kappa^{2} V(w, t)=-\frac{3}{4}\left[-2+8 w^{2}+\left(\frac{\dot{T}}{T}+\frac{2 h_{0}}{t}\right)^{2} a_{0} t^{h_{0}}+\frac{\mathrm{e}^{-w^{2} / w_{0}^{2}}}{L^{2} T}\left(\frac{\dot{T}^{2}}{T^{2}}-\frac{\ddot{T}}{T}-\frac{h_{0} \dot{T}}{t T}\right)\right] .
$$

By replacing $t$ and $w$ by $\phi$ and $\chi$, we obtain the explicit form of the potential $V(\phi, \chi)$ in terms of the scalar fields $\phi$ and $\chi$.

As another example, we may consider the de Sitter universe, $a(t)=a_{0} \mathrm{e}^{H_{0} t}$ with constants $a_{0}$ and $H_{0}$. As we saw in the previous sections, the de Sitter universe can be realized even in one scalar model but we now consider this example as a simple demonstration of the reconstruction by using two scalar fields. Because

$$
f(w, t)=L^{2} \mathrm{e}^{u(w, t)} a_{0}^{2} \mathrm{e}^{2 H_{0} t} \quad e(w, t)=L^{2} \mathrm{e}^{u(w, t)} a_{0} \mathrm{e}^{H_{0} t},
$$

we find

$$
\begin{aligned}
\kappa^{2} A(w, t) & =\frac{1}{2} \dot{u}^{2}+H_{0} \dot{u}+H_{0}^{2}-\ddot{u}, \\
\kappa^{2} B(w, t) & =\frac{3}{2} L^{-2} \mathrm{e}^{-u} \dot{u}^{\prime}, \\
\kappa^{2} C(w, t) & =-\frac{3}{2} u^{\prime \prime}-\frac{\mathrm{e}^{-u}}{2 L^{2}}\left\{\dot{u}^{2}+5 H_{0} \dot{u}+6 H_{0}^{2}+\ddot{u}\right\} .
\end{aligned}
$$

The second term of the r.h.s. in (5.13), which is proportional to $L^{-2}$ vanishes, if we choose

$$
u(w, t)=-3 H_{0} t+\log \left(-\alpha(w)+\mathrm{e}^{H_{0} t}\right)+\beta(w),
$$


where $\alpha(w)$ and $\beta(w)$ are arbitrary functions which does not depend on $t$ but only depend on $w$. If we substitute (5.14) into the expressions of $A, B$, and $C$ in (5.11), (5.12), and (5.13), respectively, we find

$$
\begin{aligned}
\kappa^{2} A(w, t) & =\frac{H_{0}^{2}}{\left(\mathrm{e}^{H_{0} t}-\alpha\right)^{2}}\left\{\mathrm{e}^{2 H_{0} t}-\frac{\alpha^{2}}{2}\right\}, \\
\kappa^{2} B(w, t) & =\frac{3}{2} L^{-2} \mathrm{e}^{-u} \frac{H_{0} \alpha^{\prime} \mathrm{e}^{H_{0} t}}{\left(\mathrm{e}^{H_{0} t}-\alpha\right)^{2}}, \\
\kappa^{2} C(w, t) & =-\frac{3}{2} \frac{\alpha^{\prime \prime}(w) \mathrm{e}^{H_{0} t}-\alpha(w) \alpha^{\prime \prime}(w)+\left(\alpha^{\prime}(w)\right)^{2}}{\left(\mathrm{e}^{H_{0} t}-\alpha\right)^{2}}-\frac{3}{2} \beta^{\prime \prime}(w) .
\end{aligned}
$$

Then by choosing $\alpha=0$ and $\beta^{\prime \prime}<0$, we find

$$
\begin{aligned}
\kappa^{2} A(w, t) & =H_{0}^{2}, \\
\kappa^{2} B(w, t) & =0, \\
\kappa^{2} C(w, t) & =-\frac{3}{2} \beta^{\prime \prime}(w) .
\end{aligned}
$$

Because $A$ and $C$ are positive definite, no ghost field appears in this model.

Thus, we reconstruct two models, corresponding to the power-law expanding universe and to the de Sitter universe. The latter has been constructed by using one scalar model as in the previous section. As we saw, however, the argument about the existence of ghost becomes rather simpler in the two scalar model than in the one scalar model. By smoothly connecting the two models corresponding to the power-law expanding universe and to the de Sitter universe, we may construct a model which unifies, inflation, matter dominant universe, and the late-time accelerating expansion of the universe.

\section{SUMMARY AND DISCUSSIONS}

By using the formulation of the reconstruction, we have found the models which have an exact solution describing the domain wall. The shape of the domain wall can be flat, de Sitter space-time, or anti-de Sitter space-time. In the domain wall solutions, there often appears ghost with negative kinetic energy. We have constructed, however, an example of the de Sitter domain wall solution without ghost, which can be a toy model of inflation. We have also investigated the localization of gravity and it has been demonstrated that the four dimensional Newton law could be reproduced.

We have also shown that a space-time, where the domain wall is the general FRW universe and the warp factor can be arbitrary, can be constructed by using the two scalar fields. It has been shown that the scalar field equations are equivalent to the Bianchi identities: $\nabla^{\mu}\left(R_{\mu \nu}-\frac{1}{2} R g_{\mu \nu}\right)=0$.

We have not, however, studied if the domain wall solution is stable or unstable. About the previous work on the stability of the domain wall, see [16].

For the check of the (in)stability, we need to consider the time-dependent perturbation from the solution. The existence of the massless graviton, which is obtained from the fluctuation of the metric, may inform that the model could be stable under the perturbation of the metric. In order to verify the stability, however, we of course need to include the perturbation of the scalar field $\varphi$, or $\phi$ and $\chi$, which could be a future work.

\section{Acknowledgments.}

We are grateful to S. D. Odintsov for the discussion when he stays in Nagoya University. S.N. is supported by

Global COE Program of Nagoya University (G07) provided by the Ministry of Education, Culture, Sports, Science \& Technology and by the JSPS Grant-in-Aid for Scientific Research (S) \# 22224003 and (C) \# 23540296.

[1] L. Randall and R. Sundrum, Phys. Rev. Lett. 83, 3370 (1999) hep-ph/9905221.

[2] L. Randall, R. Sundrum, Phys. Rev. Lett. 83, 4690-4693 (1999). hep-th/9906064.

[3] G. R. Dvali, G. Gabadadze and M. Porrati, Phys. Lett. B 485, 208 (2000) hep-th/0005016. 
[4] C. Deffayet, Phys. Lett. B 502, 199 (2001) arXiv:hep-th/0010186.

[5] C. Deffayet, G. R. Dvali and G. Gabadadze, Phys. Rev. D 65, 044023 (2002) astro-ph/0105068.

[6] S. Nojiri, S. D. Odintsov and S. Zerbini, Phys. Rev. D 62, 064006 (2000) arXiv:hep-th/0001192.

[7] S. W. Hawking, T. Hertog and H. S. Reall, Phys. Rev. D 63, 083504 (2001) arXiv:hep-th/0010232.

[8] S. Nojiri and S. D. Odintsov, Phys. Lett. B 484, 119 (2000) arXiv:hep-th/0004097.

[9] A. Lukas, B. A. Ovrut, K. S. Stelle and D. Waldram, Phys. Rev. D 59, 086001 (1999) hep-th/9803235.

[10] N. Kaloper, Phys. Rev. D 60, 123506 (1999) hep-th/9905210.

[11] H. A. Chamblin and H. S. Reall, Nucl. Phys. B 562, 133 (1999) hep-th/9903225.

[12] O. DeWolfe, D. Z. Freedman, S. S. Gubser and A. Karch, Phys. Rev. D 62, 046008 (2000) hep-th/9909134.

[13] M. Gremm, Phys. Lett. B 478 (2000) 434 hep-th/9912060.

[14] C. Csaki, J. Erlich, T. J. Hollowood and Y. Shirman, Nucl. Phys. B 581 (2000) 309 hep-th/0001033.

[15] M. Gremm, Phys. Rev. D 62 (2000) 044017 hep-th/0002040.

[16] S. Kobayashi, K. Koyama and J. Soda, Phys. Rev. D 65, 064014 (2002) hep-th/0107025.

[17] T. R. Slatyer and R. R. Volkas, JHEP 0704, 062 (2007) hep-ph/0609003.

[18] S. Nojiri and S. D. Odintsov, Phys. Rept. 505, 59 (2011) arXiv:1011.0544 [gr-qc]].

[19] K. Bamba, S. Nojiri and S. D. Odintsov, Phys. Rev. D 85, 044012 (2012) arXiv:1107.2538 [hep-th]].

[20] S. Capozziello, S. Nojiri and S. D. Odintsov, Phys. Lett. B 632, 597 (2006) arXiv:hep-th/0507182. 